\title{
Expert opinion on current and emerging treatment options in androgenetic alopecia and telogen effluvium: an Indian perspective
}

\author{
Bharat Lalwala $^{1 *}$, Siddhartha Das $^{2}$, Srinivasa Murthy ${ }^{3}$, Abir Saraswat ${ }^{4}$, U. R. Dhanalakshmi ${ }^{5}$, \\ Mukesh Girdhar $^{6}$, Sachin Dhawan ${ }^{7}$, Kamal Jhamnani ${ }^{8}$, Deepa Kanchankoti ${ }^{9}$, Sushil Pande ${ }^{10}$
}

\author{
${ }^{1}$ Lalwala Skin, Hair Transplant Centre, Surat, Gujarat, India \\ ${ }^{2}$ Dr. Siddhartha Das, Orchid Skin Clinic, Kolkata, West Bengal, India \\ ${ }^{3}$ Skin \& Cosmetology Centre, Bangalore, Karnataka, India \\ ${ }^{4}$ Indushree Skin Clinic, Lucknow, Uttar Pradesh, India \\ ${ }^{5}$ Dr. Dhanalakshmi Skin Clinic, Chennai, Tamil Nadu, India \\ ${ }^{6}$ Dr Girdhar's Skin Clinic, Karkardooma, Delhi, India \\ ${ }^{7}$ Skin n' Smiles, Gurugram, Delhi, India \\ ${ }^{8}$ Abhiridh Specialty Clinic, Secunderabad, Telangana, India \\ ${ }^{9}$ Myskin Laser Clinic, Kandivali (East), Mumbai, Maharashtra, India \\ ${ }^{10}$ Department of Dermatology, NKP Salve Institute of Medical Sciences and Lata Mangeshkar Hospital, Nagpur \\ Maharashtra, India
}

Received: 20 May 2021

Revised: 19 July 2021

Accepted: 05 August 2021

*Correspondence:

Dr. Bharat Lalwala,

E-mail: bilalwala@yahoo.com

Copyright: (C) the author(s), publisher and licensee Medip Academy. This is an open-access article distributed under the terms of the Creative Commons Attribution Non-Commercial License, which permits unrestricted non-commercial use, distribution, and reproduction in any medium, provided the original work is properly cited.

\begin{abstract}
The diagnosis and management of hair loss needs an organized and systematic approach for recognizing pattern of hair loss and identification of hair loss etiology. Early and specific diagnosis is essential to initiate appropriate treatment in the early phases of hair loss. Topical minoxidil and oral finasteride are the only approved drugs for androgenetic alopecia (AGA). Various other treatment options are widely used but have limited clinical evidence. Similarly, there are no specific treatments recommended for telogen effluvium (TE). However, the treatment may become challenging with increasing availability of new formulations and drugs with no substantial evidence to support them. Multiple focused group discussions were conducted among Indian dermatologists to gain expert opinion on appropriate management of AGA and TE in the current scenario. This article summarizes the consensus clinical viewpoints for topical and oral medications, role of nutritional supplements, and other adjunctive therapies in managing AGA and TE. The panel highlighted that the choice of treatment for AGA and TE depends on the individual hair loss pattern and response to medications. A brief discussion on the use of shampoos and procedures has also been highlighted.
\end{abstract}

Keywords: Androgenetic alopecia, Telogen effluvium, Management, Treatment, Nutritional therapy

\section{INTRODUCTION}

Hair loss is a common dermatological complaint affecting quality of life of patients. Patients usually visit the clinic with diffuse or patchy hair loss. Androgenetic alopecia (AGA), a normal physiologic variant, is the most common type of alopecia after puberty in both males and females. Telogen effluvium (TE), a non-scarring, noninflammatory alopecia, has sudden etiology and is caused by physiologic or emotional stress. ${ }^{1}$ Female pattern hair loss (FPHL) is the most common hair loss disorder in women. It may develop during adolescence and lead to 
progressive hair loss with a characteristic pattern of nonscarring diffuse alopecia. ${ }^{2}$ Proper history and physical examination help understand the hair loss pattern and determine the specific etiology. Further investigations are needed to identify causative factors or rule out or detect any underlying comorbidity. ${ }^{1}$ Good clinical decisionmaking needs better understanding of pathophysiology, identification of causal factors underlying hair loss, and a specific diagnosis. Evidence-based medicine with clinical expertise is often necessary for treatment of hair loss. ${ }^{3}$ Currently, a wide range of treatment options are available for management of hair loss, including approved evidence-based drugs and off-label medications. Additionally, various adjunctive therapies are also employed but are supported with limited clinical data. Among existing and emerging treatment options for hair loss, it is necessary to understand changing treatment trends and to identify effective and safe treatment options in the current scenario for appropriate hair loss management.

Focused group discussions among 83 dermatologists across various regions in India were conducted via teleconference. The objective of the meetings was to obtain clinical perspective of experts on the management of AGA and TE in current clinical practice. A consensus expert opinion was then derived from discussions on various aspects of treatment and management. This article was formulated using 72 published research articles, systematic reviews, and/or meta-analyses, and outlines of the consensus opinion of the experts. For literature search, PubMed and Google were accessed to identify relevant articles using Boolean operators "AND" and "OR" and /keywords like hair loss, androgenetic alopecia, telogen effluvium, female pattern hair loss, management, diagnosis, minoxidil, finasteride, nutrition, and supplements were searched.

\section{EPIDEMIOLOGY OF AGA AND TE}

\section{Prevalence of $A G A$}

The onset of AGA is estimated to be at any age following puberty and its frequency is observed to be increasing with age. 4 The prevalence of AGA is found to be highest in the Caucasian population, affecting up to $80 \%$ Caucasian men and $42 \%$ of women. ${ }^{4,5}$ In India, around $58 \%$ prevalence of AGA has been estimated in men aged 30-50 years, with type II and III as the most common presentation. ${ }^{5}$ Approximately, $6 \%-38 \%$ of healthy women report some degree of frontal and/or frontal-parietal hair loss. ${ }^{2}$

\section{Prevalence of TE}

TE is the most common concurrent hair loss condition affecting $38.6 \%$ of patients with FPHL. ${ }^{6}$ Incidence of TE and female pattern hair loss (FPHL) was found to be highest in the age group of 21-30 years, whereas chronic TE was predominant in the $30-40$-year age group. ${ }^{7}$

\section{Consensus statement 1}

The panel stated that equal proportion of males and females report with hair problems; AGA is more commonly observed in males, affecting the age group of 20-50 years. In women, the condition is often underdiagnosed; hence; lack of evidence on the same has been reported. In women, AGA is often confused with TE. FPHL is most commonly observed in obese females in mid-30s, and particularly in females with symptoms of polycystic ovarian disease (PCOD).

\section{DIAGNOSIS OF AGA AND TE}

\section{History and clinical examination}

Patient history is necessary to identify the exact cause of hair loss, AGA, or TE. The typical pattern of hair loss over frontal, parietal or vertex areas, and experience of pain or itching are imperative. Family history, history of diseases and concomitant medications, hormonal dysfunction, and dietary and lifestyle factors must be considered. ${ }^{5}$ Clinical examination of hair and scalp is performed to identify if hair loss is patterned or not. Pulltest may be performed to assess severity of hair loss. ${ }^{5}$ Trichoscopy is a useful diagnostic tool for AGA. The main features of AGA on trichoscopy are hair diameter diversity greater than $20 \%$, perifollicular pigmentation/peripilar signs, and yellow dot.5 AGA/TE wash test can be used to distinguish between AGA and TE. It is based on count of vellus and terminal telogen hair strands that are rinsed out on washing the scalp after a 5-day abstinence from washing. ${ }^{5}$

\section{Laboratory investigations}

Although laboratory investigations are not needed in AGA cases in males, prostate specific antigen test may be done before initiating finasteride in men aged $>45$ years. In women, laboratory tests are conducted to rule out underlying hormonal dysfunction as observed in polycystic ovarian disorder. Tests include free androgen index test, dehydroepiandrosterone (DHEAS) and prolactin. ${ }^{5}$ Laboratory tests like complete blood count, urine analysis, serum ferritin, T3, T4, and thyroid stimulating hormone should be performed to diagnose TE. Other less common tests include antinuclear antibody titer and serum zinc level. ${ }^{8}$

\section{Consensus statement 2}

The experts agreed that examination and detailed history are highly advised for AGA and TE. Clinical diagnosis of AGA in males involves bitemporal recession whereas in females it involves more of frontal hair thinning with a commonality in reducing diameter, miniaturization of terminal hair, and vellus hair. Tests to be performed include trichoscopy, hair pull test, and scalp biopsy. In AGA, trichoscopy findings show yellow dots, which is a distinctive feature that is absent in TE. Clinical trigger 
factors for TE include history of stress, hospitalization, dengue, any febrile illness, pre-existing disease, and history of excessive bleeding. Blood sugar levels and insulin resistance test are considered, especially in males with early onset AGA. In TE, basic set of investigations include serum ferritin test, vitamin B12, D3, hemoglobin and thyroid profile. Overall, hair loss profile investigations include hemogram, ferritin, iron stores, sex hormone binding globulin, vitamin B12, vitamin $\mathrm{D}$, PCOD, and insulin.

\section{OVERLAP OF AGA AND TE}

Identification and diagnosis may be difficult if people have hair loss due to multiple coexisting forms of alopecia. Wohltmann and Sperling reported multifactorial alopecia from single biopsy specimens in $12.5 \%$ of cases. AGA was found to be coexisting with traction alopecia, end-stage scarring alopecia, TE, and androgenetic alopecia (AA). ${ }^{9}$

\section{Consensus statement 3}

According to experts, females often present with chronic TE progressing to patterned alopecia. In $\sim 50 \%$ of females who present with PCOD, metabolic syndrome, and an unhealthy lifestyle, chronic TE progresses to FPHL. Coexistence of TE and FPHL is also prevalent in obese females and after childbirth. Use of trichoscopy in overlap cases helps to differentiate between AGA and TE. History of crash diet and keto diet is commonly identified in TE, which adversely affects hair loss and progresses to AGA. Important differentiation between AGA and TE is that thinning of hair in the occipital area is not seen in AGA.

\section{TREATMENT AND MANAGEMENT}

\section{Conventional treatment in AGA}

Among androgen-dependent agents, topical minoxidil is the only Food and Drug Administration (FDA) approved drug for management of AGA. Minoxidil 2\% and 5\% are approved for male AGA; whereas only $2 \%$ is approved for female AGA. Finasteride (1 mg once daily [OD]) is the only oral drug approved for treatment of male AGA. Dutasteride is considered a potential alternative to finasteride and is currently approved for benign prostatic hyperplasia. Clinical studies have demonstrated its efficacy and safety in treatment of male AGA. ${ }^{10}$

\section{Topical treatment in $A G A$}

\section{Topical minoxidil}

Minoxidil 2\% solution twice daily is effective for preventing progression and improving AGA in frontotemporal and vertex regions. ${ }^{4}$ In men with AGA, $5 \%$ topical minoxidil was found to be superior to $2 \%$ topical minoxidil in increasing hair growth, indicated by $45 \%$ more hair regrowth than $2 \%$ topical minoxidil at week $48 .{ }^{11}$ The efficacy of $5 \%$ solution (once daily) is observed to be comparable to $2 \%$ solution (twice daily). ${ }^{4}$ In females, $2 \%$ topical solution has demonstrated better efficacy than $1 \%$ solution. Topical minoxidil treatment should be continued for at least 6 months before assessing its efficacy. ${ }^{10}$ On getting expected results, the treatment needs to be continued to maintain efficacy. ${ }^{4}$

\section{High concentration minoxidil}

Besides the approved 2\% and 5\% topical minoxidil solutions, various concentrations of minoxidil solution in the range $2 \%-12 \%$ are available. In a 36-week study in men with AGA, Ghonemy et al observed superiority of $5 \%$ solution over $10 \%$ solution for hair growth with respect to total vertex and frontal hair mean count. Irritation was observed to be high for $10 \%$ topical minoxidil. ${ }^{12}$

\section{Vehicles of minoxidil}

Minoxidil has poor skin penetration ability; alcohol and propylene glycol facilitates solubility and uptake of minoxidil in tissues. ${ }^{13,14}$ Propylene glycol is associated with adverse reactions such as itching, irritation, scaling, redness, contact dermatitis, and dryness. ${ }^{13,15}$ Thus, alternative solvents like butylene glycol, glycerin, or polysorbate are used to avoid potential hypersensitive reactions with minoxidil, especially in propylene glycolsensitive patients. ${ }^{14,16,17}$ Foam formulation of minoxidil, which is propylene glycol-free, exhibits application benefits such as better drug penetration and ability to deliver a greater amount of the active drug at an increased rate, compared with other vehicles. ${ }^{15} \mathrm{~A}$ comparative study of 5\% minoxidil solution and 5\% minoxidil milky lotion (butylene glycol solvent) demonstrated that both formulations are equally effective and can be used as an alternative treatment for propylene glycol-sensitive patients. ${ }^{16}$

\section{Consensus statement 4}

Experts agreed that first-line topical treatment for AGA is $5 \%$ minoxidil for males and $2 \%$ minoxidil for females. Higher strengths of $10 \%$ can also be used in male patients, who do not benefit from $5 \%$ minoxidil. In addition, steroid lotions are prescribed in patients who show irritancy and flaking with minoxidil use. Flaking and irritation is often seen with $10 \%$ minoxidil because of the propylene glycol base; hence, compliance issues are observed in certain patients. The panel opined that alcohol and propylene glycol-free preparations of minoxidil are superior due to lesser irritation and itching, and thus help better patient compliance. Additionally, foams tend to liquefy and are difficult to apply. Thus, solution-based formulations can be preferred as they may be less messy than foams. 


\section{Combination therapies of minoxidil}

\section{Minoxidil and finasteride}

Topical minoxidil and finasteride combination demonstrated good hair density maintenance after initial improvement with oral finasteride. In addition, topical combination treatment was well tolerated with good compliance. ${ }^{18}$ Combination of $3 \%$ minoxidil and $0.1 \%$ finasteride lotion showed significant improvement in men with AGA on comparison with $3 \%$ minoxidil solution. ${ }^{19}$ A 12-month study in male AGA patients demonstrated better efficacy of oral finasteride and topical 5\% minoxidil combination (94\%) compared to finasteride and minoxidil monotherapies (80\% versus 59\%, respectively). ${ }^{20}$ Studies have demonstrated efficacy of topical finasteride without significant systemic adverse effects in men with AGA.19,20 Topical formulations of finasteride could minimize systemic side effects of oral finasteride. ${ }^{21}$ Suchonwanit et al demonstrated that topical combination of $0.25 \%$ finasteride and $3 \%$ minoxidil was effective in postmenopausal women with FPHL versus $3 \%$ minoxidil solution with significantly superior increase in hair diameter. ${ }^{21}$

\section{Minoxidil and peptides}

Botanical ingredients including peptides are safe alternative treatment options. ${ }^{22}$ Redensyl is a combination of four active ingredients: dihydroquercetin-glucoside (DHQG), epigallocatechin gallate glucoside (EGCG2), glycine, and zinc. DHQG and EGCG2 (two stabilized polyphenols) target and stimulate fibroblasts in the outer root sheath stem cells and dermal papilla, and glycine and zinc are required for hair metabolism. ${ }^{22}$ Capixyl, a biomimetic peptide (mixed with red clover extract), demonstrated increase in hair width and density by rebalancing anagen/telogen ratio and promoting healthy hair growth. ${ }^{23}$

\section{Minoxidil and procapil}

Procapil is a combination of three plant-derived substances, oleanolic acid (inhibits $5 \alpha 1$ and $5 \alpha 2$ reductase enzymes), apigenin for vasodilation, and glycinehistidine- lysine peptides which have pro-matrix metalloproteinase activity. ${ }^{22}$ A randomized controlled study compared combination of procapil, redensyl, and capixyl with $5 \%$ minoxidil for 24 weeks. The peptide group showed 1.5 times greater improvement versus minoxidil group. ${ }^{22}$ Additionally, better patient satisfaction was reported with topical minoxidil combined with peptides like procapil versus topical minoxidil alone. ${ }^{22}$

\section{Minoxidil and other combination therapies}

Vitamin A and its derivatives may have a significant role in growth and maintenance of hair follicles. Tretinoin has demonstrated enhancement of more dense hair in combination with minoxidil. ${ }^{24}$ Combination of minoxidil and tretinoin was observed to increase percutaneous absorption of minoxidil by 3 -fold by increasing stratum corneum permeability. ${ }^{25}$ Azelaic acid is a potent inhibitor of 5-alpha reductase and could be an effective adjunctive treatment in androgen related pathology. ${ }^{26}$

\section{Consensus statement 5}

In clinical practice, minoxidil is given with oral finasteride depending on severity of hair fall. In patients who are not comfortable with oral finasteride due to side effects, topical finasteride is considered.

Topical peptide combinations add up to the efficacy of minoxidil; procapil and capixyl potentiate 5- $\alpha$ reductase inhibition. In cases where minoxidil monotherapy needs to be avoided, minoxidil combined with peptides can be used. The combination of minoxidil, azelaic acid, and tretinoin is preferred in diffuse AGA. It can also be given to patients with seborrhea along with male pattern alopecia. Azelaic acid is a $5 \alpha$ - dihydrotestosterone (DHT) blocker, and tretinoin increases penetration and efficacy of minoxidil and dermal thickness.

\section{Topical treatment in TE}

Although there is no specific treatment for management of TE, topical minoxidil has been reported to be effective as it prolongs anagen phase. ${ }^{8}$ Amino acids or topical peptides are also indicated to provide benefits in TE. ${ }^{27} \mathrm{~A}$ range of vitamins, minerals, and botanical products for multimodal treatment of hair loss are available. ${ }^{28}$ Melatonin influences hair growth by inducing anagen phase. ${ }^{29}$ Adenosine has demonstrated an increase in growth rate of anagen hair and thick hair rate. ${ }^{30}$ Other ingredients like serum-based peptides, caffeinated preparations, and topical botanicals may also be useful. ${ }^{27}$

\section{Consensus statement 6}

The experts preferred peptides containing sera, procapil, botanicals and vitamin supplements as topical treatment for TE. Topical minoxidil can be used in case of chronic TE. Peptide formulations with multivitamins have shown good results.

\section{Oral treatment in $A G A$}

Finasteride is the first approved oral drug for treatment of men with AGA. It is considered as the most effective oral therapy for AGA based on extensive clinical data. ${ }^{31}$ Finasteride at the dose of $1 \mathrm{mg}$ per day is considered as the optimal dose and long-term use results in sustained improvement. ${ }^{32}$ Sexual side effects of finasteride were reported to occur at the rate of $2.1 \%-3.8 \%$, which was comparable to placebo. ${ }^{32}$ Overall, current literature on the safety of finasteride indicates that it is a safe drug. ${ }^{32} \mathrm{In}$ female pattern AGA, oral finasteride at doses of 2.5 $\mathrm{mg} /$ day or $5 \mathrm{mg} /$ day was effective for treatment of patterned hair loss in postmenopausal women. ${ }^{33,34}$ 


\section{Consensus statement 7}

The panel advised that in males, $1 \mathrm{mg}$ finasteride or 0.5 mg dutasteride could be considered as oral treatment. Spironolactone is useful in females who are not planning to get pregnant in the future. Also, finasteride $2.5 \mathrm{mg}$ or 5 $\mathrm{mg}$ is useful in females who have completed their family and in post-menopausal women.

\section{Oral treatment in $\mathrm{TE}$}

Hair growth is influenced by diet via complex metabolic interactions. $^{35}$ Unmet nutritional needs can be supplemented by nutritional therapy in the form of vitamins, minerals, and proteins ${ }^{8,35}$ Nutrition replacement therapy can be considered for catagen promoting deficiencies like iron, zinc, estradiol, and protein deficiency. ${ }^{8}$ Sulphur containing amino acids, cysteine and methionine, are necessary for hair building. Other amino acids like L-lysine, are important for hair shape and volume. Vitamins C, B-complex and A have an impact on state of hair. Minerals like zinc, iron, copper, selenium, magnesium, and calcium are known to influence hair growth. ${ }^{36}$ Vitamin $\mathrm{D}$ has been suggested to delay aging including hair loss. ${ }^{2}$

\section{Consensus statement 8}

According to experts, nutritional supplements, a diet rich in iron and vitamin B-12, biotin supplements, cysteine formulations and calcium supplementations must be considered in TE.

\section{Nutritional supplementation in AGA and TE}

Studies have reported a causal link between nutritional deficiencies and pathology of AGA, TE, and female pattern hair loss. ${ }^{35}$ Low serum ferritin has been associated with increased prevalence in patients with chronic TE, FPHL, AGA, and AA. Despite multiple studies, inconsistent data suggests link of iron deficiency to hair loss. Limited information exists on effects of zinc supplementation on hair growth. ${ }^{35}$ The benefit of oral supplementation with amino acids, biotin, zinc, and other micronutrients in hair loss of any origin is controversial. ${ }^{2}$ There are only a few studies and anecdotal evidences on a number of nutritional supplements for hair growth. There is a lack of good quality randomized controlled trials to support the claims.

\section{Consensus statement 9}

Experts emphasized that nutritional supplementation plays a secondary role in AGA treatment and is usually given for 3-6 months. Multivitamins and iron supplements are necessary for TE. The panel agreed that there can be deficiencies in otherwise healthy participants with hair loss irrespective of the type of alopecia. In people with nutritional deficiencies, supplementation might be useful in preventing hair loss.

\section{Novel formulations in AGA and TE}

\section{Topical caffeine-based formulations}

Caffeine promotes cell proliferation by stimulating cell metabolism. ${ }^{37}$ Topical application of caffeine improves barrier function and follicular penetration and stimulates and promotes hair growth. ${ }^{37}$

\section{Oral minoxidil therapy}

Oral minoxidil is used off-label for AGA and FPHL, but it is associated with dose-related side effects. ${ }^{2}$ Oral minoxidil at a dose of $5 \mathrm{mg}$ daily was effective with acceptable safety profile in male patients with AGA. ${ }^{38}$ Clinical studies and case reports have shown therapeutic benefits with oral minoxidil for hair loss treatment; however, optimal dosing of oral minoxidil needs to be determined. ${ }^{39}$

\section{Supplements}

Dietary insufficiency of vitamins, minerals, or quality proteins may correlate with clinical and subclinical hair loss. There could be increased nutrient loss or poor nutrient absorption contributing to dietary insufficiency. ${ }^{40}$ Epigallocatechin gallate, a major constituent of polyphenols, has been shown to stimulate growth of hair follicles by proliferative and apoptotic effects of dermal papilla cells. It might be useful in prevention or treatment of AGA. ${ }^{41}$ Combined administration of capsaicin and isoflavone might increase insulin-like growth factor-1 (IGF1) production in hair follicles, which plays an important role in hair growth. Subjects with alopecia were observed to have increased plasma levels of IGF1 at 5 months of oral administration of capsaicin (6 mg/day) and isoflavone $(75 \mathrm{mg} /$ day $){ }^{42}$ Hair shedding was significantly reduced in the first 3-6 months after daily oral consumption of marine protein-based supplement. ${ }^{40}$

\section{Cyclical therapy}

Cyclical nutritional therapy it maintains low gradient in circulation and leads to more efficient absorption and complete utilization. It also helps reduce dosage and improve efficiency of nutrients. It enables inclusion of a broad range of essential vitamins, minerals, amino acids, fatty acids, coenzymes, and supportive nutrients in therapy. ${ }^{43}$

\section{Consensus statement 10}

The experts highlighted good penetration properties of caffeine-based formulations. However, lotions or shampoos with caffeine have shown to increase anagen phase. Data on oral minoxidil data are limited.

Protein supplements (tablet/powder) can be used as a supplementation. Experts expressed preference for plantbased protein supplements. Additionally, supplements 
with antioxidant ingredients like green tea extract can also be used.

The panel suggested that ingredients that show an interaction with each other can be spaced out in two different formulations in a day. Supplementation could be given as a comprehensive nutritional intervention with ingredients spaced out (day and night regimen).

\section{Treatment for AGA and TE overlap}

Very few studies have reported multiple forms of alopecia in patients with hair loss. Overlap of clinical presentation makes identification and diagnosis challenging. ${ }^{9}$

\section{Consensus statement 11}

The panel highlighted that in AGA and TE overlap cases, topical minoxidil is initiated as the first choice of treatment. Oral finasteride in males and females can also be considered. However, usually topical minoxidil or minoxidil with peptides is considered in females due to possible adverse events of finasteride. Nutritional supplements are given as required.

\section{Use of cosmeceuticals in AGA and TE}

\section{Shampoos}

Besides treating dandruff and seborrheic dermatitis, zincbased shampoos have shown reduction in hair loss. They may be included as an integral part of treatment regimen for hair loss, even in absence of scalp pathologies. ${ }^{44} \mathrm{TE}$ was controlled by ketoconazole, piroctone olamine, and zinc pyrithione shampoos at $1 \%$ concentration in men with TE related to androgenic alopecia associated with dandruff. Additionally, ketoconazole and piroctone olamine increased mean hair shaft thickness and decreased sebum output. ${ }^{45}$ Vigorous shampoo application may damage hair and thus, must be avoided. ${ }^{27}$

\section{Hair styling products}

Cosmetic products minimize hair fiber breakage, improve hair volume, or conceal visible bald scalp, and thus, improve overall appearance. Products providing lubrication also help in minimizing hair friction and may provide temporary, external 'gluing' to prevent damage to uplifted cuticles, thus reducing hair breakage. Along with shampoos, other topical products can add bulk to the hair fiber surface, lift/hold the hair and help mask visible scalp. ${ }^{46}$ Oil can be used as a pre-wash conditioner to protect cuticles and make hair smooth and shiny. ${ }^{27}$

\section{Consensus statement 12}

The panel stated that generally shampoos are not prescribed as part of treatment procedure. However, if the patient insists, sulphate-free, mild shampoos, at neutral/acidic $\mathrm{pH}$, having fewer preservatives, perfumes or chemicals are preferred. Shampoos containing antiandrogenic and anti-inflammatory ingredients are preferred.

Experts advised to avoid any hair styling products during the treatment period. If required, leave-on conditioners or deep conditioning masks can be used for dry and frizzy hair to improve hair manageability. Vitamin E-based serums, oils, and tocopherol oil may work well. Hair oil can be avoided if the patient is on minoxidil or has seborrhea or any scalp issues. The experts expressed need for a good pharmaceutical preparation for hair grooming, which could be used along with minoxidil.

\section{Sensitive scalp in AGA and TE}

Seborrheic dermatitis (SD) was the most common disease associated with AGA in men (51.2\%) and women $(45.7 \%) .{ }^{47} \mathrm{SD}$ can be related to increased production of DHT in affected areas. Increased DHT, a key factor in seborrheic dermatitis and AGA, stimulates the sebaceous gland. ${ }^{67}$ Sensitive scalp may get worsened and be triggered by hair loss. ${ }^{48}$

\section{Consensus statement 13}

Experts emphasized that in cases of sensitive scalp, conditions like iron deficiency, inflammation, or alopecia, areata need to be ruled out. It may also be necessary to investigate neuropsychiatric association of sensitive scalp. Alcohol-free minoxidil formulations or peptide preparations could be used in patients with scalp sensitivity.

\section{Role of hair camouflage in AGA and TE}

Camouflage options for patients who suffer from hair loss include full or partial wigs, hair extensions, concealing powders and sprays, surgical tattoos, and hair transplants. Natural wigs use real human hair fibers and give natural appearance, but are expensive and need maintenance. Synthetic wigs are easy to maintain and less expensive. Topical hair fibers made of wool or rice keratin, rayon, or human hair must be applied daily. Temporary and daily application camouflage options are pigmented concealing powders, lotions, and sprays. Powder cakes are circular concealing disks that can be applied to the scalp with a sponge. Camouflage lotions and sprays are applied to limit color contrast between existing hair and scalp. Permanent hair camouflaging options include hair transplantation and micropigmentation. ${ }^{49}$

\section{Consensus statement 14}

According to the experts, hair camouflage options are beneficial for patients who present with extensive alopecia areata, alopecia universalis, and hair patches, and patients who shaved off their hair for surgery. Pigmented concealing powders, lotions, and sprays can 
be used. Hair fibers can be used in patients who have undergone hair transplantation or patients with early AGA. Using hair patches or transplantation is mainly based on patients' expectation. Many patients prefer concealers due to associated social stigma and anxiety.

\section{Procedures in AGA and TE}

Mesotherapy, a non-invasive technique, is expected to restore abnormal physiology causing alopecia by stimulating various biological responses via injecting active substances (e.g. vitamins, medications, and plant extracts) into the scalp, just below the epidermis using superficial microinjections. There is variable and limited data available to support use of mesotherapy for alopecia treatment. Microneedling pierces stratum corneum and creates numerous transient microchannels over the applied surface without damaging the epidermis. It induces collagen synthesis, stimulates stem cells and growth factors, and increases blood flow to hair follicles. There is an increased clinical interest for microneedling in treating different types of alopecia. Platelet-rich plasma enhances proliferation of dermal papilla cells and prevents cell apoptosis, thereby promoting cell growth survival of hair follicles. It has demonstrated to be an effective tool as an alternative treatment for alopecia. Lasers or light sources are also reported to improve texture and quality of hair and promote hair growth. Although safe and effective, large placebo-controlled studies are needed. ${ }^{50}$

\section{Consensus statement 15}

Experts highlighted that platelet-rich plasma therapy is often considered as a supportive or add-on therapy or if there is no/less response observed with minoxidil. It is also used in combination with other therapies like microneedling for AGA treatment. Laser light therapy is difficult to implement in clinical practice; however, it has shown positive effects.

\section{CONCLUSION}

For specific diagnosis of hair loss, detailed history, clinical pattern of hair loss, diagnostic tools like trichoscopy, triggering factors, and information regarding underlying diseases or nutritional deficiencies are essential. While experts provided comprehensive, clinical views for appropriate management of AGA and TE, it was agreed that individual clinical pattern and patient compliance play an equally important role in the management plan. Use of alcohol-free or propyleneglycol free minoxidil solution (5\% for males and $2 \%$ for females) can be helpful in lowering irritation. Combination therapies with minoxidil could be considered to increase effectiveness of minoxidil. Topical peptide formulations along with multivitamins or oral iron, vitamin B-12, biotin supplements, cysteine formulations, and calcium supplementations must be considered in TE. Lastly, mild and sulfate-free shampoos with acidic $\mathrm{pH}$ containing anti-androgenic and antiinflammatory ingredients could be used, if necessary. Although, substantial evidence is still lacking for several drugs and supplements, this consensus provides valuable insights for current management of AGA and TE in India.

\section{ACKNOWLEDGEMENTS}

The authors thank Scientimed Solutions Pvt. Ltd. for assisting in development of this manuscript.

\section{Funding: No funding sources \\ Conflict of interest: None declared \\ Ethical approval: Not required}

\section{REFERENCES}

1. Phillips TG, Slomiany WP, Allison R. Hair loss: Common causes and treatment. Am Fam Physician. 2017;96:371-8.

2. Fabbrocini G, Cantelli M, Masarà A, Annunziata MC, Marasca C, Cacciapuoti S. Female pattern hair loss: A clinical, pathophysiologic, and therapeutic review. Int J Womens Dermatol. 2018;4:203-11.

3. Truieb RM. The difficult hair loss patient: a particular challenge. Int J Trichology. 2013;5:110-4.

4. Kanti V, Messenger A, Dobos G, Reygagne P, Finner A, Blumeyer A, et al. Evidence-based (S3) guideline for the treatment of androgenetic alopecia in women and in men-short version. J Eur Acad Dermatol Venereol. 2018;32:11-22.

5. Kaliyadan F, Nambiar A, Vijayaraghavan S. Androgenetic alopecia: an update. Indian $\mathbf{J}$ Dermatol Venereol Leprol. 2013;79:613-25.

6. Siah TW, Muir-Green L, Shapiro J. Female pattern hair loss: A retrospective study in a tertiary referral center. Int J Trichology. 2016;8:57-61.

7. Malkud S. A hospital-based study to determine causes of diffuse hair loss in women. J Clin Diagn Res. 2015;9:WC01-4.

8. Malkud S. Telogen effluvium: A review. J Clin Diagn Res. 2015;9:WE01-3.

9. Wohltmann WE, Sperling L. Histopathologic diagnosis of multifactorial alopecia. J Cutan Pathol. 2016;43:483-91.

10. Varothai S, Bergfeld WF. Androgenetic alopecia: An evidence-based treatment update. Am J Clin Dermatol. 2014;15:217-30.

11. Olsen EA, Dunlap FE, Funicella T, Koperski JA, Swinehart JM, Tschen EH, et al. A randomized clinical trial of $5 \%$ topical minoxidil versus $2 \%$ topical minoxidil and placebo in the treatment of androgenetic alopecia in men. J Am Acad Dermatol. 2002; 47:377-85.

12. Ghonemy S, Alarawi A, Bessar H. Efficacy and safety of a new $10 \%$ topical minoxidil versus $5 \%$ topical minoxidil and placebo in the treatment of male androgenetic alopecia: a trichoscopic evaluation. J Dermatol Treat. 2021;2:236-41. 
13. Purnak T, Senel E, Sahin C. Liquid formulation of minoxidil versus its foam formulation. Indian $\mathrm{J}$ Dermatol. 2011;56:462.

14. Rossi A, Cantisani C, Melis L, Iorio A, Scali E, Calvieri S. Minoxidil use in dermatology, side effects and recent patents. Recent Pat Inflamm Allergy Drug Discov 2012;6:130-6

15. Gogtay JA, Panda M. Minoxidil topical foam: A new kid on the block. Int J Trichology. 2009;1:142.

16. Thuangtong R, Thanomkitti K, Suvanasuthi S. A comparative study between the efficacy and safety of $5 \%$ minoxidil solution and $5 \%$ minoxidil milky lotion in the treatment of male androgenic alopecia. J Med Assoc Thai. 2017;100:70-7.

17. Suchonwanit P, Thammarucha S, Leerunyakul K. Minoxidil and its use in hair disorders: a review. Drug Des Devel Ther. 2019;13:2777-86.

18. Chandrashekar BS, Nandhini T, Vasanth V, Sriram R, Navale S. Topical minoxidil fortified with finasteride: An account of maintenance of hair density after replacing oral finasteride. Indian Dermatol Online J. 2015;6:17-20.

19. Tanglertsampan C. Efficacy and safety of $3 \%$ minoxidil versus combined $3 \%$ minoxidil / $0.1 \%$ finasteride in male pattern hair loss: a randomized, double-blind, comparative study. J Med Assoc Thai. 2012;95:1312-16.

20. Hu R, Xu F, Sheng Y, Qi S, Han Y, Miao Y, et al. Combined treatment with oral finasteride and topical minoxidil in male androgenetic alopecia: a randomized and comparative study in Chinese patients. Dermatol Ther. 2015;28:303-8.

21. Suchonwanit $\mathrm{P}$, Iamsumang W, Rojhirunsakool S. Efficacy of topical combination of $0.25 \%$ finasteride and $3 \%$ minoxidil versus $3 \%$ minoxidil solution in female pattern hair loss: A randomized, doubleblind, controlled study. Am J Clin Dermatol. 2019;20:147-53.

22. Karaca N, Akpolat ND. A comparative study between topical 5\% minoxidil and topical "redensyl, capixyl, and procapil" combination in men with androgenetic alopecia. J Cosmo Trichol. 2019;5:1-7.

23. Loing E, Lachance R, Ollier V, Hocquaux M. A new strategy to modulate alopecia using a combination of two specific and unique ingredients. J Cosmet Sci. 2013;64:45-58.

24. Kwon OS, Pyo HK, Oh YJ, Han JH, Lee SR, Chung $\mathrm{JH}$, et al. Promotive effect of minoxidil combined with all-trans retinoic acid (tretinoin) on human hair growth in vitro. J Korean Med Sci. 2007;22:283-9.

25. Ferry JJ, Forbes KK, VanderLugt JT, Szpunar GJ. Influence of tretinoin on the percutaneous absorption of minoxidil from an aqueous topical solution. Clin Pharmacol Ther. 1990;47:439-46.

26. Stamatiadis D, Bulteau-Portois MC, Mowszowicz I. Inhibition of 5 alpha-reductase activity in human skin by zinc and azelaic acid. $\mathrm{Br} \mathrm{J}$ Dermatol 1988;119:627-32

27. Mysore V, Parthasaradhi A, Kharkar RD, Ghoshal AK, Ganjoo A, Ravichandran G, et al. Expert consensus on the management of Telogen Effluvium in India. Int J Trichology. 2019;11:107-12.

28. Hosking AM, Juhasz M, Mesinkovska AN. Complementary and alternative treatments for alopecia: A comprehensive review. Skin Appendage Disord. 2019;5:72-89.

29. Fischer TW, Burmeister G, Schmidt HW, Elsner P. Melatonin increases anagen hair rate in women with androgenetic alopecia or diffuse alopecia: results of a pilot randomized controlled trial. Br J Dermatol. 2004;150:341-5.

30. Oura H, Iino M, Nakazawa Y, Tajima M, Ideta R, Nakaya $\mathrm{Y}$, et al. Adenosine increases anagen hair growth and thick hairs in Japanese women with female pattern hair loss: a pilot, double-blind, randomized, placebo-controlled trial. J Dermatol. 2008;35:763-7.

31. Sorbellini E, Pinto D, Marzani B, Rinaldi F. Drug treatment for androgenetic alopecia: First Italian questionnaire survey on what dermatologists think about finasteride. Dermatol Ther (Heidelb). 2018;8:259-67.

32. Mysore V, Shashikumar BM. Guidelines on the use of finasteride in androgenetic alopecia. Indian $\mathrm{J}$ Dermatol Venereol Leprol. 2016;82:128-34.

33. Iorizzo M, Vincenzi C, Voudouris S, Piraccini BM, Tosti A. Finasteride treatment of female pattern hair loss. Arch Dermatol. 2006;142:298-302.

34. Oliveira-Soares R, E Silva JM, Correia MP, André MC. Finasteride $5 \mathrm{mg} /$ day treatment of patterned hair loss in normo-androgenetic postmenopausal women. Int J Trichology. 2013;5:22-5.

35. Guo EL, Katta R. Diet and hair loss: effects of nutrient deficiency and supplement use. Dermatol Pract Concept. 2017;7:1-10.

36. Goluch-Koniuszy ZS. Nutrition of women with hair loss problem during the period of menopause. Prz Menopauzalny. 2016;15:56-61.

37. Bansal M, Manchanda K, Pandey SS. Role of caffeine in the management of androgenetic alopecia. Int J Trichology. 2012;4:185-86.

38. Jimenez-Cauhe J, Saceda-Corralo D, RodriguesBarata R, Hermosa-Gelbard A, Moreno-Arrones OM, Fernandez-Nieto D, et al. Effectiveness and safety of low-dose oral minoxidil in male androgenetic alopecia. J Am Acad Dermatol. 2019;81:648-9.

39. Beach RA. Case series of oral minoxidil for androgenetic and traction alopecia: Tolerability \& the five C's of oral therapy. Dermatol Ther. 2018;31:e12707.

40. Rizer RL, Stephens TJ, Herndon JH, Sperber BR, Murphy J, Ablon GR. A marine protein-based dietary supplement for subclinical hair thinning/loss: results of a multisite, double-blind, placebo-controlled clinical trial. Int $\mathbf{J}$ Trichology. 2015;7:156-66.

41. Kwon OS, Han JH, Yoo HG, Chung JH, Cho KH, Eun $\mathrm{HC}$, et al. Human hair growth enhancement in 
vitro by green tea epigallocatechin-3-gallate (EGCG). Phytomedicine. 2007;14:551-55.

42. Harada N, Okajima $\mathrm{K}$, Arai M, Kurihara $\mathrm{H}$, Nakagata N. Administration of capsaicin and isoflavone promotes hair growth by increasing insulin-like growth factor-I production in mice and in humans with alopecia. Growth Horm IGF Res. 2007; 17:408-15.

43. Rajput R. The Concept of Cyclical Nutritional Therapy for Hair Growth which can be Applied for Wellness. J Nutr Food Sci. 2017;7:615.

44. Trüeb RM, Henry JP, Davis MG, Schwartz JR. Scalp Condition Impacts Hair Growth and Retention via Oxidative Stress. Int J Trichol. 2018;10:262-70.

45. Piérard-Franchimont C, Goffin V, Henry F, Uhoda I, Braham C, Piérard GE. Nudging hair shedding by antidandruff shampoos. A comparison of $1 \%$ ketoconazole, $1 \%$ piroctone olamine and $1 \%$ zinc pyrithione formulations. Int $\mathrm{J}$ Cosmet Sci. 2002;24:249-56.
46. Sinclair R, Patel M, Dawson Jr TL, Yazdabadi A, Yip L, Perez A, et al. Hair loss in women: medical and cosmetic approaches to increase scalp hair fullness. Br J Dermatol. 2011;165:12-8.

47. Jang WS, Son IP, Yeo IK, Park KY, Li K, Kim BJ, et al. The annual changes of clinical manifestation of androgenetic alopecia clinic in Korean males and females: a outpatient-based study. Ann Dermatol. 2013;25:181-8.

48. Godse K, Zawar V. Sensitive scalp. Int J Trichology. 2012;4:102-4.

49. Saed S, Ibrahim O, Bergfeld WF. Hair camouflage: A comprehensive review. Int J Womens Dermatol. 2017; (1):S75-80.

50. Kartal SP, Altunel C, Gencler B. Cosmetic procedures in the treatment of alopecia. Hair and scalp disorders. 2017;3:317.

Cite this article as: Lalwala B, Das S, Murthy S, Saraswat A, Dhanalakshmi UR, Girdhar M et al. Expert opinion on current and emerging treatment options in androgenetic alopecia and telogen effluvium: an Indian perspective Int J Res Dermatol. 2022;8:152-60. 